\title{
Analysis of the molecular mechanisms of Pinus sylvestris and Hordeum vulgare adaptation to the action of low doses of ionizing radiation
}

\author{
P. Volkova ${ }^{1}$, G. Duarte ${ }^{2}$, E. Kazakova ${ }^{1 *}$ \\ ${ }^{1}$ Russian Institute of Radiology and Agroecology, Obninsk, Russia \\ ${ }^{2}$ Institute Jean-Pierre Bourgin, Versailles, France \\ *e-mail: alvaly@mail.ru
}

Key words: adaptation, irradiation, low doses, gene expression, Pinus sylvestris, Hordeum vulgare

Motivation and Aim: The revealing of molecular mechanisms for the formation of adaptive plant responses to low-intensity stress factors (including low doses of ionizing radiation) is an important problem in modern biology. In order to clarify the mechanisms of adaptation of plants to the action of low-dose radiation we are conducting two experiments: (1) the study of chronically irradiated populations of Pinus sylvestris from the exclusion zone of accident at the Chernobyl nuclear power plant, and (2) on Hordeum vulgare plants growing from gamma-irradiated seeds. The results obtained can be applied in the field of radiation protection of biota and genetic engineering of crops. Methods and Algorithms: For the identification of the genes involved in the formation of the response to chronic irradiation (annual dose up to $130 \mathrm{mGy}$ ) in populations of P. sylvestris, a transcriptome analysis using high-throughput RNA sequencing was performed. Analysis of expression of genes involved in the response of seedlings of $H$. vulgare on irradiation of seeds in doses of $0,20,100 \mathrm{~Gy}$ is performed using DNAmicroarrays (Agilent). The validation of the results in both experiments will be done by analyzing the expression of selected candidate genes by RT-PCR.

Results: After analysis of transcripts in chronically irradiated populations of $P$. sylvestris we identified the main groups of genes responding to chronic exposure. These genes code the transcription factors, components of the antioxidant system, membrane proteins, histones, genes associated with the biosynthesis of ABA, genes maintaining work of chloroplasts and activity of transposons. There was no differential expression of genes associated with repair processes of DNA damage [1]. Candidate genes for the further validation were chosen. The analysis of the transcriptome of $H$. vulgare is currently under way.

Conclusion: The populations of $P$. sylvestris respond to chronic radiation exposure by the differential expression of hundreds of genes, the analysis of which has helped to approach the understanding of the basis of plant reactions to chronic low-dose irradiation under natural conditions. It is also assumed that $H$. vulgare transcriptome analysis will identify the genes involved in the adaptive response of plants to low-dose irradiation in the laboratory conditions (in particular, the formation of the effect of radiation stimulation). Acknowledgements: Supported by RSF (14-14-00666), by RSF (17-74-10028).

\section{References}

1. Duarte G.T. et al. (2018) Radioactive contamination in the Chernobyl zone promotes changes in gene expression in gymnosperms. Nature Ecology Evolution (under review). 